\title{
Triggering of Neoclassical Tearing Mode by Error Field Penetration
}

\author{
Seiya NISHIMURA and Ryusuke NUMATA ${ }^{1)}$ \\ Department of Electric and Electronic Engineering, Hosei University, Koganei 184-8584, Japan \\ ${ }^{1)}$ Graduate School of Simulation Studies, University of Hyogo, Kobe 650-0047, Japan
}

(Received 27 June 2018 / Accepted 22 July 2018)

\begin{abstract}
We conduct the first simulation study of the neoclassical tearing mode (NTM) triggered by error field penetration. A series of processes including the error field penetration, formation of seed islands, and the triggering of the NTM occurs, when the plasma flow and the electron diamagnetic drift approximately cancel out each other. The excited NTM is the born-locked mode, which is locked from the beginning. In the case where the plasma flow and the electron diamagnetic drift completely cancel out each other, a vacuum island width necessary for triggering the born-locked NTM is much smaller than a seed island width necessary for triggering the original NTM. This tendency is consistent with a theoretical prediction. Thus, whether the born-locked NTM is excited depends on both the plasma flow velocity and the error field amplitude.
\end{abstract}

(c) 2018 The Japan Society of Plasma Science and Nuclear Fusion Research

Keywords: neoclassical tearing mode, error field, plasma flow, perturbed bootstrap current, forced magnetic reconnection

DOI: $10.1585 /$ pfr.13.1203104

In tokamaks, magnetic islands driven by the neoclassical tearing mode (NTM) reduce the achievable $\beta$ value, and the locking of the magnetic island rotation by resistive wall or error fields occasionally triggers disruptions of plasma discharges. To avoid locked magnetic islands is a particularly important technical issue. The NTM is a nonlinear instability destabilized by the perturbed bootstrap current [1]. For triggering the NTM, finite-amplitude perturbations called seed islands are necessary. The error field is caused by positioning errors of the main coil system. The error field induces the forced magnetic reconnection [2], even when the NTM is stable. The forced magnetic reconnection is prevented by plasma flows, i.e., the error field is shielded by plasma flows [3]. The formation of magnetic islands due to error fields is called the error field penetration. In experiments in JET, born-locked modes, which are the NTM locked at its onset, have been observed, where the error field penetration triggers the NTM [4-6]. Using a modified Rutherford equation, possibility of excitation of the born-locked NTM in ITER is pointed out [1]. However, the seed island formation by error field penetration and the consequent evolution of the NTM have not been fully investigated. In preceding works, it is found that the error field penetration occurs, when the plasma flow and the electron diamagnetic drift approximately cancel out each other [7-9]. In those works, an effect of the perturbed bootstrap current is not taken into account. However, the stability of the NTM becomes a problem in high $\beta$ regime. In this paper, we consider the error field penetration as a seeding mechanism of the NTM.
We consider tokamak plasmas with the minor radius $a$, the major radius $R_{0}$, and the toroidal magnetic field $B_{0}$. We introduce a reduced set of two-fluid equations [10], where the bootstrap current is phenomenologically introduced. We assume that the quasi-neutral density is constant in time and space, and the pressure is proportional to the temperature. The normalized reduced two-fluid equations are

$$
\begin{aligned}
& \frac{\partial U}{\partial t}+[\phi+\delta \tau p, U]=\delta \tau\left[\nabla_{\perp} \phi ; \nabla_{\perp} p\right]+\nabla_{\|} j_{\|} \\
& +\mu \nabla_{\perp}^{2} U, \\
& \frac{\partial \psi}{\partial t}=-\nabla_{\|}(\phi-\delta p)-\eta_{\|}\left(\tilde{j}_{\|}-\tilde{j}_{\mathrm{bs}}\right), \\
& \frac{\partial p}{\partial t}+[\phi, p]=\epsilon^{2} \chi_{\|} \nabla_{\|}^{2} p+\chi_{\perp} \nabla_{\perp}^{2} p,
\end{aligned}
$$

with $U=\nabla_{\perp}^{2} \phi$ and $j_{\|}=-\nabla_{\perp}^{2} \psi$, where $U$ is the vorticity, $\phi$ is the electrostatic potential, $j_{\|}$is the parallel current density, $\tilde{j}_{\|}$is the parallel current density perturbation, $\tilde{j}_{\mathrm{bs}}$ is the bootstrap current perturbation, $\psi$ is the magnetic flux, $p$ is the electron pressure, $\mu$ is the perpendicular viscosity coefficient, $\eta_{\|}$is the parallel resistivity, $\chi_{\|}$is the parallel thermal diffusivity, $\chi_{\|}$is the perpendicular thermal diffusivity, $\delta$ is the ion skin depth, $\epsilon=a / R_{0}, \tau$ is the ratio of the ion temperature to the electron tempearture, $\left\{\nabla_{\perp}, \nabla_{\|}\right\}$are the perpendicular and parallel spatial differential operators, respectively, and the bracket with the semicolon in Eq. (1) is the spatial differential operator defined in Ref. [10]. The time is normalized by $\tau_{\mathrm{A}}=R_{0} / v_{\mathrm{A}}$, where $v_{\mathrm{A}}$ is the Alfvén velocity, and $\left\{\nabla_{\perp}, \nabla_{\|}\right\}$are normalized by $a$ and $R_{0}$, respectively. Our simulation set up is shown as follows. Assuming single-helicity modes resonant at the rational surface, 


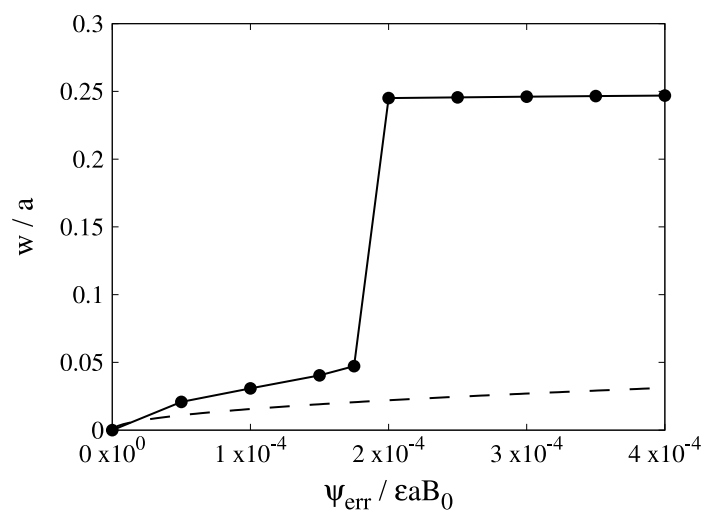

Fig. 1 Dependence of saturated island widths on $\psi_{\text {err }}$. The vacuum island width is shown by a dashed line.

we only consider spacial profile of variables in the twodimensional coordinates $(x, y)$, where $x$ is the radial position and $y$ is the poloidal position. The box size in the $x-y$ space is $L_{x} \times L_{y}=1 \times \pi$, where the radial and poloidal boundaries are located at $x=0,1$ and $y=0, \pi$, respectively. The bootstrap current perturbation is given by $\tilde{j}_{\mathrm{bs}}=$ $-\left(f_{\mathrm{bs}} q_{\mathrm{s}} / 2 \sqrt{\epsilon}\right) \partial_{x} \tilde{p}$, where $f_{\mathrm{bs}}=1.46$ and $q_{\mathrm{s}}$ is the safety factor at the rational surface. The equilibrium quantities are given by $U_{0}=0, \phi_{0}=u_{0} x, \psi_{0}=\ln \left[\cosh \left(x-x_{\mathrm{s}}\right)\right], p_{0}=$ $(\beta / \epsilon)\left[1-\left(x-x_{\mathrm{s}}\right) /\left(1-x_{\mathrm{s}}\right)\right]$, where $x_{\mathrm{s}}=0.5$ is the radial position of the rational surface, and $u_{0}$ is the equilibrium poloidal flow velocity constant in space. Boundary conditions of perturbation in the $y$ direction are periodic, and those in the $x$ direction are fixed. The error field is applied in terms of the magnetic flux perturbation as $\left.\tilde{\psi}(t, x, y)\right|_{x=1}=\psi_{\text {err }} \cos \left(k_{y} y\right)$, where $\psi_{\text {err }}$ is the amplitude, $m_{0}$ is the poloidal mode number of the error field, and $k_{y}=$ $2 \pi m_{0} / L_{y}$. In addition, the relative magnitude of the error field is evaluated as $B_{\mathrm{r}} / B_{0}=\epsilon k_{y} \psi_{\text {err }}$, where $B_{\mathrm{r}}$ is the radial component of the error field at $x=1$. In simulations, the following parameters are fixed: $\tau=1, \beta=0.02, \delta=0.02$, $\epsilon=0.3, q_{\mathrm{s}}=2, m_{0}=2, \tau=1, \eta_{\|}=10^{-5}, \chi_{\perp}=10^{-5}$, $\mu=\chi_{\perp} / 4$, and $\epsilon^{2} \chi_{\|}=1$. For consistency with the singlehelicity assumption, only perturbations with poloidal mode numbers $m=0,2,4, \ldots$ are considered. The tearing mode stability parameter for the $m=2$ mode is $\Delta^{\prime}=-7.86$. In addition, the electron diamagnetic drift velocity is given by $v_{* \mathrm{e}}=-\delta\left(d p_{0} / d x\right)=2 \delta \beta / \epsilon=2.67 \times 10^{-3}$. In simulations, $u_{0}$ and $\psi_{\text {err }}$ are used as control parameters.

First, nonlinear stability analysis of the NTM is performed. Using $u_{0}=0$ and $\psi_{\text {err }}=0$, time evolution of magnetic island widths for various seed island widths $w_{0}$ is simulated. It is found that the magnetic island grows to a large size $w_{\text {sat }}=0.24$, only when the seed island width exceed a critical value $w_{0}^{\text {crit }}=0.08$, and damps in other cases. Next, we consider cases where the NTM and the error field coexist. In the following part of this paper, $w_{0}$ is sufficiently small. In simulations, $u_{0}=-v_{* \mathrm{e}}=-2.67 \times 10^{-3}$ is chosen. Figure 1 shows the error field amplitude de-

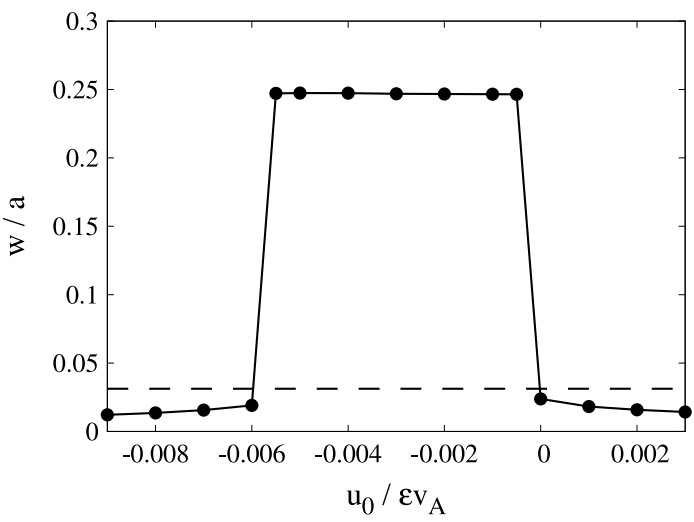

Fig. 2 Dependence of saturated island widths on $u_{0}$. The vacuum island width is shown by a dashed line.

pendence of saturated widths of magnetic islands which are locked from the beginning. It is shown that sudden transition occurs between $\psi_{\text {err }}=1.75 \times 10^{-4}$ and $\psi_{\text {err }}=2 \times 10^{-4}$, and the born-locked NTM is triggered in the large $\psi_{\text {err }}$ regime. Note that the critical vacuum island width for triggering the born-locked NTM is about $w_{\mathrm{v}}^{\text {crit }}=0.02$, which is much smaller than $w_{0}^{\text {crit }}$. A theoretical analysis based on the modified Rutherford equation predicts $w_{\mathrm{v}}^{\text {crit }} / w_{0}^{\text {crit }}=2 /(3 \sqrt{3})=0.384 \ldots[1]$, which roughly agrees with the simulation result $w_{\mathrm{v}}^{\text {crit }} / w_{0}^{\text {crit }}=0.25$. Finally, we consider shielding effects of plasma flows in the presence of the electron diamagnetic drift. Figure 2 shows the dependence of saturated magnetic island widths on the value of $u_{0}$ for $\psi_{\mathrm{err}}=4 \times 10^{-4}$. In the regime not far from $u_{0}=-v_{* \mathrm{e}}=-2.67 \times 10^{-3}$, the born-locked NTM is triggered, while, suppression of the born-locked NTM following the error field shielding occurs in other regime.

In summary, we confirmed that the born-locked NTM is triggered, when the error field amplitude exceeds a critical value, and when plasma flows and the electron diamagnetic drift approximately cancel out each other.

This work is based on our presentation in the 45th European Physical Society Conference on Plasma Physics. This work was supported by JSPS KAKENHI Grant Number $16 \mathrm{~K} 18337$. The computation in this work has been done using the facilities of the Center for Cooperative Work on Computational Science, University of Hyogo. S. N. would like to acknowledge a Collaborative Research Program of Research Institute for Applied Mechanics, Kyushu University.

[1] R. Fitzpatrick, Phys. Plasmas 2, 825 (1995).

[2] H.S. Hahm and R.M. Kulsrud, Phys. Fluids 28, 2412 (1985).

[3] R. Fitzpatrick, Nucl. Fusion 33, 1049 (1993).

[4] G.M. Fishpool and P.S. Haynes, Nucl. Fusion 34, 109 (1994).

[5] R.J. Buttery, M. De' Benedetti, T.C. Hender and B.J.D. Tubbing, Nucl. Fusion 40, 807 (2000). 
[6] R.J. Buttery and T.C. Hender, Fusion Sci. Technol. 53, 1080 (2008).

[7] Y. Kikuchi, M.F.M. de Bock, K.H. Finken, M. Jakubowski, R. Jaspers, H.R. Koslowski, A. Kraemer-Flecken, M. Lehnen, Y. Liang, G. Matsunaga, D. Reiser, R.C. Wolf, O. Zimmermann and TEXTOR-Team, Phys. Rev. Lett. 97, 085003 (2006).
[8] Q. Yu, S. Günter, Y. Kikuchi and K.H. Finken, Nucl. Fusion 48, 024007 (2008).

[9] F. Militello and F.L. Waelbroeck, Nucl. Fusion 49, 065018 (2009).

[10] R.D. Hazeltine, M. Kotschenreuter and P.J. Morrison, Phys. Fluids 28, 2466 (1985). 